\title{
Stochastic master equation for a probed system in a cavity
}

\author{
Anne E. B. Nielsen and Klaus Mølmer \\ Lundbeck Foundation Theoretical Center for Quantum System Research, \\ Department of Physics and Astronomy, University of Aarhus, DK-8000 Arhus C, Denmark
}

(Dated: October 29, 2018)

\begin{abstract}
We present a detailed derivation of the stochastic master equation determining the time evolution of the state of a general quantum system, which is placed inside a cavity and subjected to indirect measurements by monitoring the state of electromagnetic radiation transmitted through the cavity. The derivation is based on the physics involved and the final result is stated in terms of the physical parameters of the setup. To illustrate the predictions contained in the equation, we solve it analytically for a specific system, and we demonstrate quantum jumps and freezing of the internal coherent dynamics of the system as a result of continued measurements.

PACS numbers: 42.50.Dv, 03.65.Wj, 03.67.-a
\end{abstract}

\section{INTRODUCTION}

The time evolution of the state of an isolated quantum mechanical system is governed by the Schrödinger equation. If the system is subjected to a measurement, the state of the system collapses onto an eigenstate of the measured observable, and the measurement outcome is the corresponding eigenvalue. Instead of performing a measurement directly on the system, one may also allow the system to interact with an auxiliary system, which is then subjected to a measurement. This measurement procedure reveals only partial information on the state of the original system and is very useful in protocols to prepare a system in a certain quantum mechanical state and to achieve quantum nondemolition measurements of an observable. The system could for instance be clouds of atoms or few atoms that are probed with a beam of light 1, 2, 3, 4] or an electromagnetic field mode in a cavity probed with a beam of atoms [5].

When a system interacts with the surroundings, it is not practical to keep track of the complete state of both system and surroundings, and instead the degrees of freedom of the surroundings are traced out. In this case the state of the system is no longer pure and must be described by a density operator, whose time evolution is determined by the master equation. For a system subjected to indirect measurements, the time evolution depends on the actual outcomes of the measurements, and since these are probabilistic in nature, they are handled mathematically by introducing stochastic variables into the master equation. This allows one to calculate both the state of the system conditioned on a given set of measurement outcomes (a given realization of the stochastic variables) and the probability to observe that particular sequence of measurement results [6].

The stochastic master equation is often derived in very general settings, and it has been shown that all master equations must be on the so-called Lindblad form [7] in order to preserve complete positivity of the density operator. The generality is, however, achieved at the cost of introduction of abstract measurement operators and measurement strengths. In contrast, in the present pa- per we assume a concrete measurement scheme and derive the stochastic master equation directly from known physical interactions. The system under consideration is placed inside a cavity and probed with electromagnetic radiation by sending photons into the cavity from one side and performing homodyne measurements on the field leaving the cavity on the opposite side. The purpose of the cavity is to reflect the probe light several times before it is detected whereby the effective interaction strength between the light field and the system is increased as we shall demonstrate below.

Probing of atomic systems by their effect on the transmission properties of an optical cavity have a long history in quantum optics. Early studies focused on the field-atom dynamics, leading, for instance, to the normalmode splitting of the transmission resonance [8] and on photon statistics [9], while more recent work has shown the possibility to observe the spatial motion of individual atoms trapped inside the cavity by the probing beam itself [10, 11]. See also the work on optically transported or guided atoms [12, 13, 14]. More recently, optical cavities have been introduced in experiments to probe BoseEinstein condensates [15, 16, 17].

A stochastic master equation for a setup involving a cavity and a homodyne detector has been derived using a rather different approach in Ref. [18]. In that paper, however, the aim was not to use an auxiliary system to perform indirect measurements but to determine the time evolution of the field in a cavity, when the light leaking out of the cavity is subjected to measurements, and thus the probing light and the probed system, which are crucial ingredients in the present paper, were not included in the analysis.

The paper is structured as follows. In Sec. III we describe the physical setup under consideration and discuss the time evolution of the density matrix. If the dynamics is slow compared to the round trip time of light in the cavity, we can use a continuous description and derive the stochastic master equation in Sec. III. In Sec. IV we comment further on the derived equation, and in Sec. $\mathrm{V}$ we apply it to specific systems to illustrate explicitly how the state of the system is gradually collapsed 
by the measurements and to investigate how the relative strength of the probing and the coherent dynamics of the system influences the time evolution. Sec. VI concludes the paper.

\section{MODEL OF THE PROBING PROCEDURE}

The probing procedure applied is shown schematically in Fig. 1. The system is placed inside a four sided ring cavity. The probe light enters the cavity from the left, it interacts with the system, and eventually it leaks out of the cavity, where the transmitted light is subjected to a balanced homodyne measurement. In order to describe the quantum state of the light field we divide all the light beams into small segments of duration $d t$ as illustrated in Fig. 2 and treat each segment as a single mode. This is valid provided $d t$ is chosen sufficiently small. The state of the light and the system is then specified as the collective state of all the light modes and the system. To avoid keeping track of an enormous number of modes, we assume that the probe light is in a coherent state before it enters into the cavity. The modes of the probe beam that have not yet reached the cavity are then in a product state, and their time evolution is independent of the dynamics inside the cavity and the outcome of the homodyne measurements. These modes can consequently be excluded from the analysis until the time, when they arrive at the cavity. The vacuum field incident on the beam splitter $\mathrm{BS}_{2}$ in Fig. 1 and the coherent state local oscillator field are also product states, and again it is sufficient to consider the modes that are currently entering. The modes that are detected in the homodyne detector are traced out after the measurements, and the unobserved modes that leave the cavity at $\mathrm{BS}_{1}$ are also traced out in each time step. The number of active modes is then constant in time, and in the following we denote the density operator of the light modes inside the cavity, the light modes between the cavity and the detectors, and the system at time $t$ by $\rho(t)$. We note that the analysis can also be carried out for a squeezed input field by including an optical parametric oscillator in front of the cavity in Fig. 1. For a suitable theoretical description of the optical parametric oscillator see Ref. [19].

To determine the time evolution of $\rho(t)$ we express $\rho(t+d t)$ in terms of $\rho(t)$. The relevant modes are the modes included in $\rho(t)$ and the mode of the probe field, the vacuum state mode, and the local oscillator mode that reach beam splitters $\mathrm{BS}_{1}, \mathrm{BS}_{2}$, and $\mathrm{BS}_{3}$, respectively, in the time interval between $t$ and $t+d t$, and the state of these modes is $\rho(t) \otimes \rho_{\text {in }}(t) \otimes|0\rangle\langle 0|\otimes| \alpha\rangle\langle\alpha|$. In the time between $t$ and $t+d t$ several interactions take place. The state of the two modes that hit the beam splitter $\mathrm{BS}_{1}$ are mixed, and this process is described by the unitary operator $U_{1}$. Similar transformations, described by the operators $U_{2}$ and $U_{3}$, occur at beam splitters $\mathrm{BS}_{2}$ and $\mathrm{BS}_{3}$. The system itself undergoes evolution during $d t$ and the light mode that passes the system at time $t$ interacts

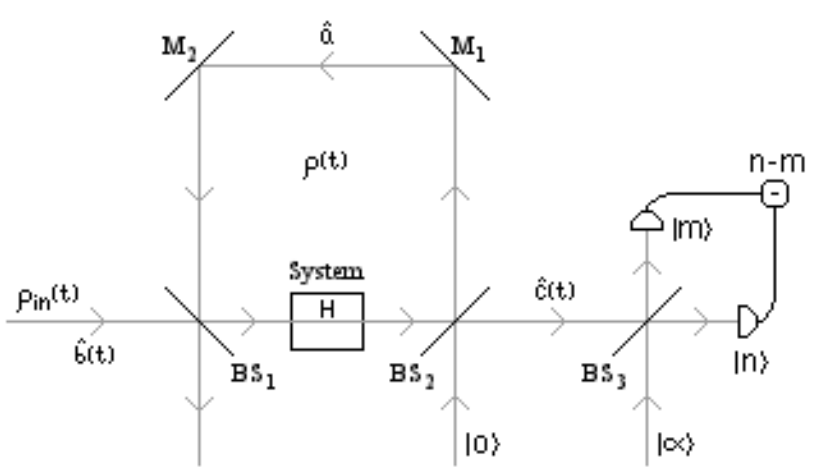

FIG. 1: Model of the setup. The cavity consists of the two mirrors $\mathrm{M}_{1}$ and $\mathrm{M}_{2}$ and the two beam splitters $\mathrm{BS}_{1}$ and $\mathrm{BS}_{2}$. The probe light $\rho_{\text {in }}(t)$ enters the cavity through $\mathrm{BS}_{1}$. The cavity field interacts with the system and leaks out of the cavity through $\mathrm{BS}_{1}$ and $\mathrm{BS}_{2}(|0\rangle$ is a vacuum state). The light transmitted through the cavity is subjected to balanced homodyne detection. $\mathrm{BS}_{3}$ is a $50: 50$ beam splitter, $|\alpha\rangle$ is a strong local oscillator, and the two detectors register $n$ and $m$ photons, respectively. The measurement readout is $k=n-m$.

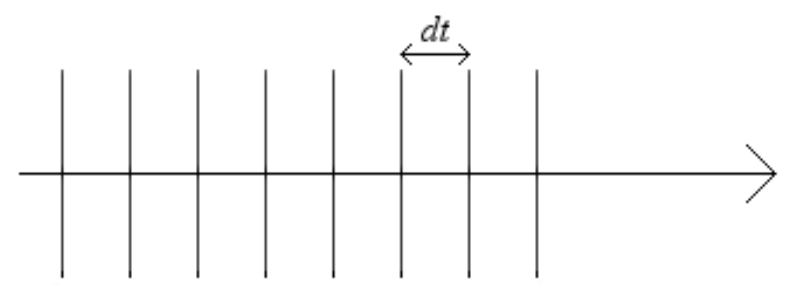

FIG. 2: The light beams are divided into temporal modes of (infinitesimal) duration $d t$.

with it. The corresponding infinitesimal time evolution operator is $U_{H}=1-i H d t / \hbar$, where $H=H_{\mathrm{sys}}+H_{I}$ is the sum of the system Hamiltonian $H_{\text {sys }}$ and the Hamiltonian $H_{I}$ for the interaction between the system and the light field. We note that the system could be subjected to manipulations that depend on the state of the system and light field and such feedback terms would be included in $H_{\text {sys }}$. In the following we disregard decay of the system, since it does not add any interesting points to the analysis, and it is easily included by adding a term to the final equation (see for instance [6] for a derivation of the relevant term for a system consisting of a single two-level atom). If the system is an extended object that interacts strongly and, for example, depletes the light field, it would be relevant to slice the system into small pieces and consider interactions between the system and several light modes in each time step, but we ignore such complications here. We shall also assume that the mirrors $\mathrm{M}_{1}$ and $\mathrm{M}_{2}$ induce phase shifts of $\pi / 2$, which are taken into account through the operators $U_{M_{1}}$ and $U_{M_{2}}$. Finally, the two modes that hit the detectors in the time inter- 
val $d t$ are projected on photon number states $|n\rangle$ and $|m\rangle$, respectively. Even though the detectors are able to resolve the exact number of photons microscopically, the only macroscopically available measurement readout is the difference $k=n-m$ between the number of detected photons in the two detectors. We thus have to average over all possible values of $n$ and $m$ that lead to the observed value of $k$. Putting all the transformations together, we obtain the density operator at time $t+d t$ conditioned on the measurement of a difference of $k$ photons in the interval from $t$ to $t+d t$

$$
\begin{array}{r}
\rho(t+d t)=\frac{1}{P_{k}} \operatorname{Tr}_{1}\left(\sum_{m}\langle m+k|\langle m| U_{3} U_{1} U_{M_{2}}\right. \\
U_{M_{1}} U_{2} U_{H} \rho(t) \otimes \rho_{\text {in }}(t) \otimes|0\rangle\langle 0|\otimes| \alpha\rangle\langle\alpha| \\
\left.U_{H}^{\dagger} U_{2}^{\dagger} U_{M_{1}}^{\dagger} U_{M_{2}}^{\dagger} U_{1}^{\dagger} U_{3}^{\dagger}|m\rangle|m+k\rangle\right),
\end{array}
$$

where $P_{k}$ is the probability, determined from the normalization of $\rho(t+d t)$, to obtain the measurement outcome $k$, and $\operatorname{Tr}_{1}$ denotes the trace over the unobserved mode leaving the cavity at beam splitter $\mathrm{BS}_{1}$.

The time evolution of $\rho(t)$ is completely specified by Eq. (1), and in principle one can start with a given initial state and iterate (1) on a computer. This task does, however, soon become unwieldy due to the large number of modes that are involved. In the next section we show how Eq. (11) can be simplified considerably and rewritten as a stochastic differential equation by invoking a few assumptions.

\section{DERIVATION OF THE STOCHASTIC MASTER EQUATION}

The key assumption in the following derivation is that all changes of the state of the light field takes place on a time scale that is large compared to the round trip time $\tau$ of light in the cavity, which is valid if the cavity is sufficiently small. In this case the temporal width of the light modes can be chosen as large as $\tau$, and, in particular, the field inside the cavity can be treated as a single mode. The assumption requires that the coupling between the system and the light field is not too large, since, for instance, the fraction of the light absorbed or emitted during a single round trip must be small. The finesse of the cavity must also be high and the total number of photons in a segment of duration $\tau$ of the input beam must be small in order to avoid that a significant number of photons leak out of or enter into the cavity within a time $\tau$. Finally, variations in, for instance, the input field or the interactions between the system and the light field that take place on a time scale small compared to $\tau$ are not allowed, since they will be smeared out. Actually, the input field typically has such fast variations because the size of the cavity is large compared to the wavelength of the input light, but, provided the frequency of the radiation is not too far from a cavity resonance, this problem is easily circumvented by moving into a frame rotating with the relevant resonance frequency of the cavity, and in the following all frequencies are to be measured relative to the cavity resonance. With these requirements we can treat $\tau$ as an infinitesimal quantity, and below we shall denote $\tau$ by $d t$. For simplicity, we shall also assume that the homodyne detector is placed within a distance $c \tau$ from $\mathrm{BS}_{2}$, where $c$ is the speed of light, since in that case $\rho(t)$ includes only the state of the system and a single mode of the light field (the cavity mode).

When $\tau$ is infinitesimal, the transmission $t_{1}^{2}\left(t_{2}^{2}\right)$ of beam splitter $\mathrm{BS}_{1}\left(\mathrm{BS}_{2}\right)$ must also be infinitesimal, and we define $\kappa_{i}$ by

$$
t_{i}^{2}=\kappa_{i} d t, \quad i=1,2 .
$$

This allows us to rewrite the unitary operator

$$
U_{1}=e^{i \frac{\pi}{2} \hat{a}^{\dagger} \hat{a}} e^{i \frac{\pi}{2} \hat{b}^{\dagger} \hat{b}} e^{-i \tan ^{-1}\left(\frac{t_{1}}{r_{1}}\right)\left(\hat{a}^{\dagger} \hat{b}+\hat{a} \hat{b}^{\dagger}\right)},
$$

representing $\mathrm{BS}_{1}$, as

$$
\begin{aligned}
U_{1}=e^{i \frac{\pi}{2} \hat{a}^{\dagger} \hat{a}} e^{i \frac{\pi}{2} \hat{b}^{\dagger} \hat{b}} & \\
& \left(1-i t_{1}\left(\hat{a}^{\dagger} \hat{b}+\hat{a} \hat{b}^{\dagger}\right)-\frac{1}{2} t_{1}^{2}\left(\hat{a}^{\dagger} \hat{b}+\hat{a} \hat{b}^{\dagger}\right)^{2}\right)
\end{aligned}
$$

to first order in $d t$, where $r_{1}^{2}$ is the reflectivity of $\mathrm{BS}_{1}, \hat{a}$ is the field annihilation operator of the cavity field, and $\hat{b}$ is the field annihilation operator of the mode of width $d t$ of the input field that arrives at the beam splitter at time $t$. Note that (3) has been chosen such that after the interaction, the $\hat{a}$-mode is still the cavity mode, while the $\hat{b}$-mode is the mode leaving the cavity. Similarly, we have

$$
\begin{aligned}
U_{2}=e^{i \frac{\pi}{2} \hat{a}^{\dagger} \hat{a}} e^{i \frac{\pi}{2} \hat{c}^{\dagger} \hat{c}} & \left(1-i t_{2}\left(\hat{a}^{\dagger} \hat{c}+\hat{a} \hat{c}^{\dagger}\right)-\frac{1}{2} t_{2}^{2}\left(\hat{a}^{\dagger} \hat{c}+\hat{a} \hat{c}^{\dagger}\right)^{2}\right) \\
& (15
\end{aligned}
$$

where $\hat{c}$ is the field annihilation operator of the mode leaving the cavity at $\mathrm{BS}_{2}$. The operators $U_{M_{1}}$ and $U_{M_{2}}$ both act on the cavity mode and thus

$$
U_{M_{1}}=U_{M_{2}}=e^{i \frac{\pi}{2} \hat{a}^{\dagger} \hat{a}} .
$$

Since the number of photons in a segment of duration $\tau$ of the input beam is assumed to be much smaller than one, we can write

$$
\begin{aligned}
\rho_{\mathrm{in}}(t)= & c_{\mathrm{in}, 00}|0\rangle\left\langle 0\left|+c_{\mathrm{in}, 10}\right| 1\right\rangle\left\langle 0\left|+c_{\mathrm{in}, 01}\right| 0\right\rangle\langle 1| \\
& +c_{\mathrm{in}, 11}|1\rangle\left\langle 1\left|+c_{\mathrm{in}, 20}\right| 2\right\rangle\left\langle 0\left|+c_{\mathrm{in}, 02}\right| 0\right\rangle\langle 2|
\end{aligned}
$$

to first order in $d t$, where $c_{\mathrm{in}, 00}=1-c_{\mathrm{in}, 11}$ is of order unity, $c_{\mathrm{in}, 10}=c_{\mathrm{in}, 01}^{*}$ is of order $\sqrt{d t}$, and $c_{\mathrm{in}, 11}$ and $c_{\mathrm{in}, 20}=c_{\mathrm{in}, 02}^{*}$ are of order $d t$. 
Putting the relation $U_{H}=1-i H d t / \hbar$ and Eqs. (4), (5), (6), and (7) into Eq. (11), we find

$$
\begin{array}{r}
\rho(t+d t)=\frac{1}{P_{k}}\left(u_{00} \rho(t)-u_{00} \frac{i}{\hbar}[H, \rho(t)] d t+u_{00} t_{1}\right. \\
\left(\left[\hat{a}^{\dagger}, \rho(t)\right] \operatorname{Tr}\left(\hat{b} \rho_{i n}(t)\right)-[\hat{a}, \rho(t)] \operatorname{Tr}\left(\rho_{i n}(t) \hat{b}^{\dagger}\right)\right) \\
+u_{10} t_{2} \hat{a} \rho(t)+u_{01} t_{2} \rho(t) \hat{a}^{\dagger} \\
+\frac{1}{2} u_{00} t_{1}^{2}\left(-\hat{a}^{\dagger} \hat{a} \rho(t)-\rho(t) \hat{a}^{\dagger} \hat{a}+2 \hat{a} \rho(t) \hat{a}^{\dagger}\right) \\
+\frac{1}{2} t_{2}^{2}\left(-u_{00} \hat{a}^{\dagger} \hat{a} \rho(t)-u_{00} \rho(t) \hat{a}^{\dagger} \hat{a}+2 u_{11} \hat{a} \rho(t) \hat{a}^{\dagger}\right. \\
\left.\left.+\sqrt{2} u_{20} \hat{a}^{2} \rho(t)+\sqrt{2} u_{02} \rho(t)\left(\hat{a}^{\dagger}\right)^{2}\right)\right)
\end{array}
$$

where

$$
u_{p q} \equiv \sum_{m}\left\langle m+k\left|\left\langle m\left|U_{3}\right| \alpha\right\rangle\right| p\right\rangle\left\langle q\left|\left\langle\alpha\left|U_{3}^{\dagger}\right| m\right\rangle\right| m+k\right\rangle
$$

and $|p\rangle$ and $|q\rangle$ are photon number states. For a strong local oscillator $\left(|\alpha|^{2} \gg 1\right)$ we can apply the approximation

$$
\frac{1}{n !} \mu^{n} e^{-\mu} \approx \frac{1}{\sqrt{2 \pi \mu}} e^{-\frac{(n-\mu)^{2}}{2 \mu}}, \quad \mu \equiv \frac{|\alpha|^{2}}{2}
$$

and turn the sum in Eq. (9) into an integral, which leads to $\left(\alpha=|\alpha| e^{i \phi}\right)$

$$
\begin{aligned}
& u_{00}=\frac{1}{2 \sqrt{\pi \mu}} \exp \left(-\frac{k^{2}}{4 \mu}\right), \\
& u_{10}=\frac{-i k e^{-i \phi}}{\sqrt{2 \mu}} \frac{1}{2 \sqrt{\pi \mu}} \exp \left(-\frac{k^{2}}{4 \mu}\right), \\
& u_{20}=-\frac{\left(k^{2}-2 \mu\right) e^{-2 i \phi}}{2 \sqrt{2} \mu} \frac{1}{2 \sqrt{\pi \mu}} \exp \left(-\frac{k^{2}}{4 \mu}\right), \\
& u_{11}=\frac{k^{2}}{2 \mu} \frac{1}{2 \sqrt{\pi \mu}} \exp \left(-\frac{k^{2}}{4 \mu}\right) .
\end{aligned}
$$

From the normalization of Eq. (8) we find the probability to obtain the measurement outcome $k$

$$
\begin{aligned}
& P_{k}=\frac{1}{2 \sqrt{\pi \mu}} \exp \left(-\frac{k^{2}}{4 \mu}\right)\left(1-\frac{i k e^{-i \phi}}{\sqrt{2 \mu}} t_{2} \operatorname{Tr}(\hat{a} \rho(t))\right. \\
& +\frac{i k e^{i \phi}}{\sqrt{2 \mu}} t_{2} \operatorname{Tr}\left(\rho(t) \hat{a}^{\dagger}\right)+\frac{1}{2} t_{2}^{2}\left(\frac{k^{2}}{2 \mu}-1\right)\left(2 \operatorname{Tr}\left(\hat{a} \rho(t) \hat{a}^{\dagger}\right)\right. \\
& \left.\left.\quad-e^{-2 i \phi} \operatorname{Tr}\left(\hat{a}^{2} \rho(t)\right)-e^{2 i \phi} \operatorname{Tr}\left(\rho(t)\left(\hat{a}^{\dagger}\right)^{2}\right)\right)\right)
\end{aligned}
$$

Comparing this to

$$
\begin{aligned}
& \frac{1}{2 \sqrt{\pi(\mu+\epsilon)}} \exp \left(-\frac{(k-\delta)^{2}}{4(\mu+\epsilon)}\right) \approx \\
& \frac{1}{2 \sqrt{\pi \mu}} \exp \left(-\frac{k^{2}}{4 \mu}\right)\left(1+\frac{k \delta}{2 \mu}+\left(\frac{k^{2}}{2 \mu}-1\right) \frac{\epsilon}{2 \mu}\right)
\end{aligned}
$$

for $\delta \ll k$ and $\epsilon \ll \mu$, it is apparent that $P_{k}$, to first order in $d t$, is a Gaussian distribution. $\delta$ is of order $\sqrt{d t}$, and $\epsilon$ is of order $d t$, so to order $\sqrt{d t}$ we can replace $k / \sqrt{2 \mu}$ by

$$
\frac{\hat{k}}{\sqrt{2 \mu}}=-i e^{-i \phi} t_{2} \operatorname{Tr}(\hat{a} \rho(t))+i e^{i \phi} t_{2} \operatorname{Tr}\left(\rho(t) \hat{a}^{\dagger}\right)+\frac{d \hat{W}}{\sqrt{d t}},
$$

where $d \hat{W}$ is a stochastic variable that has a Gaussian probability density distribution with zero mean value and variance $d t$, and we have put a hat on $k$ to emphasize that $\hat{k}$ is now to be regarded as a stochastic variable that assumes the value $k$ with probability $P_{k}$. From the Ito calculus rule $d \hat{W}^{2}=d t$ (see [6]) it furthermore follows that

$$
\hat{k}^{2}=2 \mu
$$

to zeroth order in $d t$. Inserting Eqs. (2), (11), (12), (13), (14), (15), (17), (18) and

$$
\operatorname{Tr}\left(\hat{b} \rho_{\mathrm{in}}(t)\right)=\operatorname{Tr}\left(\rho_{\mathrm{in}}(t) \hat{b}^{\dagger}\right)^{*}=c_{\mathrm{in}, 10} \equiv \beta \sqrt{d t}
$$

into Eq. (8) we finally obtain the stochastic master equation in the form

$$
\begin{aligned}
& \rho(t+d t)=\rho(t)-\frac{i}{\hbar}[H, \rho(t)] d t \\
&+ \sqrt{\kappa_{1}}\left[\hat{a}^{\dagger}, \rho(t)\right] \beta d t-\sqrt{\kappa_{1}}[\hat{a}, \rho(t)] \beta^{*} d t \\
&- i e^{-i \phi} \sqrt{\kappa_{2}}(\hat{a} \rho(t)-\operatorname{Tr}(\hat{a} \rho(t)) \rho(t)) d \hat{W} \\
&+ i e^{i \phi} \sqrt{\kappa_{2}}\left(\rho(t) \hat{a}^{\dagger}-\operatorname{Tr}\left(\rho(t) \hat{a}^{\dagger}\right) \rho(t)\right) d \hat{W} \\
&+\frac{1}{2}\left(\kappa_{1}+\kappa_{2}\right)\left(-\hat{a}^{\dagger} \hat{a} \rho(t)-\rho(t) \hat{a}^{\dagger} \hat{a}+2 \hat{a} \rho(t) \hat{a}^{\dagger}\right) d t
\end{aligned}
$$

The second term on the right hand side represents the evolution due to the system Hamiltonian and the interaction between the system and the radiation, the third and fourth terms arise due to the feeding of probe light into the cavity at $\mathrm{BS}_{1}$, the fifth and sixth terms include the knowledge obtained from the homodyne measurements, and the seventh term describes the decay of the cavity mode due to transmission through $\mathrm{BS}_{1}$ and $\mathrm{BS}_{2}$. We note that the approximations leading to Eq. (20) can be stated more precisely as $|\alpha|^{2} \gg 1, \kappa_{1} \tau \ll 1, \kappa_{2} \tau \ll 1$, $\sqrt{\kappa_{1}}|\beta| \tau \ll 1$, and $\left|\left\langle\psi_{1}|H| \psi_{2}\right\rangle\right| \tau / \hbar \ll 1$, where $\left|\psi_{1}\right\rangle$ and $\left|\psi_{2}\right\rangle$ represent arbitrary state kets. It is allowed that $\beta$ and $H$ are time dependent, but the variation within a time interval of length $\tau$ should be small.

\section{FURTHER REMARKS}

So far we have assumed a lossless setup, but it is easy to incorporate effects of losses. To account for losses in the light field, we only need to replace the perfect mirror M2 by a partially transmitting beam splitter and include a beam splitter in front of the ideal homodyne detector with transmissivity $\eta=\eta_{D} \eta_{P}$, where $\eta_{D}$ is the efficiency 
of the detector and $1-\eta_{P}$ is the propagation loss between the cavity and the detector, and in this case Eq. (20) generalizes to

$$
\begin{aligned}
\rho(t+ & d t)=\rho(t)-\frac{i}{\hbar}[H, \rho(t)] d t \\
& +\sqrt{\kappa_{1}}\left[\hat{a}^{\dagger}, \rho(t)\right] \beta d t-\sqrt{\kappa_{1}}[\hat{a}, \rho(t)] \beta^{*} d t \\
& -i e^{-i \phi} \sqrt{\eta \kappa_{2}}(\hat{a} \rho(t)-\operatorname{Tr}(\hat{a} \rho(t)) \rho(t)) d \hat{W} \\
+ & i e^{i \phi} \sqrt{\eta \kappa_{2}}\left(\rho(t) \hat{a}^{\dagger}-\operatorname{Tr}\left(\rho(t) \hat{a}^{\dagger}\right) \rho(t)\right) d \hat{W} \\
& +\frac{1}{2} \kappa\left(-\hat{a}^{\dagger} \hat{a} \rho(t)-\rho(t) \hat{a}^{\dagger} \hat{a}+2 \hat{a} \rho(t) \hat{a}^{\dagger}\right) d t
\end{aligned}
$$

where $\kappa \equiv \kappa_{1}+\kappa_{2}+\kappa_{L}$, and $\kappa_{L}$, defined in analogy to Eq. (2), is the decay rate due to intra cavity loss. It is also possible to account for loss and decoherence of the quantum system in the cavity by introducing appropriate damping terms in Eq. (21).

If there is no decay of the system and no cavity loss and all light emerging from inside the cavity is detected with unit efficiency detectors, the dynamics will preserve the purity of an initially pure state of the atoms and the cavity field, and the stochastic master equation can be rewritten as a stochastic Schrödinger equation. This is convenient since it is significantly easier to propagate a wave function in time than a density operator. The situation can be achieved with the setup in Fig. 1 by taking the limit $\kappa_{1} \rightarrow 0$ and $|\beta| \rightarrow \infty$ while $\sqrt{\kappa_{1}}|\beta| \tau$ is kept small compared to unity. Alternatively one could measure both the light reflected and transmitted from the cavity or replace $\mathrm{BS}_{2}$ by a perfectly reflecting mirror and subject the light leaving the cavity at $\mathrm{BS}_{1}$ to homodyne detection. In the latter case the stochastic master equation is given by Eq. (20) with $\kappa_{2}$ and $\kappa_{1}+\kappa_{2}$ replaced by $\kappa_{1}$ and $\phi$ replaced by $\phi+\pi / 2$, and the stochastic Schrödinger equation reads

$$
\begin{aligned}
|\psi(t+d t)\rangle= & \left(1-\frac{i}{\hbar} H d t+\beta \sqrt{\kappa_{1}} \hat{a}^{\dagger} d t-\beta^{*} \sqrt{\kappa_{1}} \hat{a} d t\right. \\
- & -e^{-i \phi} \sqrt{\kappa_{1}}(\hat{a}-\langle\hat{a}\rangle) d W \\
- & \left.\frac{\kappa_{1}}{2}\left(\hat{a}^{\dagger} \hat{a}-2 \hat{a}\left\langle\hat{a}^{\dagger}\right\rangle+\langle\hat{a}\rangle\left\langle\hat{a}^{\dagger}\right\rangle\right) d t\right)|\psi(t)\rangle,
\end{aligned}
$$

where $\langle\cdot\rangle$ denotes expectation value.

Equation (21) is a nonlinear equation since $\operatorname{Tr}(\hat{a} \rho(t))$ and $\operatorname{Tr}\left(\rho(t) \hat{a}^{\dagger}\right)$ depend on the state $\rho(t)$. In general, $H$ may also depend on $\rho(t)$ (for instance if a state dependent feedback is applied), but in the special case of a state independent Hamiltonian, it is possible to transform Eq. (21) into a linear equation by application of the method presented in Ref. [6]. The nonlinear terms in Eq. (21) appear due to the first two terms on the right hand side of Eq. (17) and due to the normalization of the state. If we simply remove the factor $1 / P_{k}$ in Eq. (8), the norm of $\rho(t+d t)$ is the probability to obtain the measurement outcome $k$ for the time interval between $t$ and $t+d t$. Furthermore, the right hand side of the master equation is determined completely by the value $k$ assumed by $\hat{k}$ (or equivalently by the value $d W$ assumed by $d \hat{W}$ ) and is thus not changed if the probability density distribution of $d \hat{W}$ is changed. Only the probability to obtain the value of $d \hat{W}$ that leads to a specific value of $\hat{k}$ is changed. We can thus change the probability density distribution of $d \hat{W}$ provided we accept that the probability to obtain the state $\rho(t+d t)$ at time $t+d t$ is the norm of the state and not the probability to obtain the required value of $d \hat{W}$. We exploit this freedom to define the new stochastic variable $d \hat{y}$ by

$$
\frac{\hat{k}}{\sqrt{2 \mu}}=\frac{d \hat{y}}{\sqrt{d t}}
$$

and assume that the probability density distribution of $d \hat{y}$ is a Gaussian distribution with zero mean value and variance $d t$. If we omit the factor $1 / P_{k}$ in Eq. (8) and insert Eqs. (11), (12), (13), (14), and (23), we obtain a linear equation in $\rho(t)$. The trace of $\rho(t+d t)$ integrated over all possible realizations of $\hat{k}$ is unity, but since $\hat{k}$ is expressed in terms of $d \hat{y}$, we would like the trace of $\rho(t+d t)$ integrated over all possible realizations of $d \hat{y}$ to be unity, and we thus multiply the right hand side by $\sqrt{2 \mu / d t}$. Finally, to ensure that $\rho(t+d t)$ approaches $\rho(t)$ in the limit, where $d t$ approaches zero, we divide the right hand side by $\exp \left(-k^{2} /(4 \mu)\right) / \sqrt{2 \pi d t}$, which is precisely the Gaussian probability density $P_{d y}$ for $d \hat{y}$. With these changes Eq. (21) reduces to

$$
\begin{aligned}
\rho(t+d t) & =\rho(t)-\frac{i}{\hbar}[H, \rho(t)] d t \\
+ & \sqrt{\kappa_{1}}\left[\hat{a}^{\dagger}, \rho(t)\right] \beta d t-\sqrt{\kappa_{1}}[\hat{a}, \rho(t)] \beta^{*} d t \\
& -i \sqrt{\eta \kappa_{2}}\left(\hat{a} \rho(t) e^{-i \phi}-\rho(t) \hat{a}^{\dagger} e^{i \phi}\right) d \hat{y} \\
+ & \frac{1}{2} \kappa\left(-\hat{a}^{\dagger} \hat{a} \rho(t)-\rho(t) \hat{a}^{\dagger} \hat{a}+2 \hat{a} \rho(t) \hat{a}^{\dagger}\right) d t
\end{aligned}
$$

Due to the division by $P_{d y}$, the probability density to obtain a given state $\rho(t+d t)$ at time $t+d t$ is now the product of the norm of $\rho(t+d t)$ and the probability density $P_{d y}$ to obtain the required value of $d \hat{y}$. Since the stochastic variables corresponding to different time steps are independent and since Eq. (24) is linear in $\rho(t)$, the probability density to obtain the specific state after $N$ time steps is simply the probability $\prod_{i=1}^{N} P_{d y_{i}}$ to obtain the realizations $d y_{1}, d y_{2}, \ldots, d y_{N}$ of the stochastic variables multiplied by the norm of the state obtained from this realization summed over all realizations that lead to the desired state. In particular, if the state after $N$ time steps only depends on the sum $\hat{y}=\sum_{i=1}^{N} d \hat{y}_{i}$, then the probability density to obtain this state is

$$
P=\frac{1}{\sqrt{2 \pi N d t}} \exp \left(-\frac{y^{2}}{2 N d t}\right) \operatorname{Tr}(\rho(t+N d t)) .
$$

In the preceding section we have assumed that the transmitted light is observed by a homodyne detector, but a similar derivation may be carried out for an 
avalanche photo diode detector, which projects the infinitesimal modes of the transmitted light on either vacuum or a single photon state, and for completeness we state the result

$$
\begin{aligned}
\rho(t+d t) & =\rho(t)-\frac{i}{\hbar}[H, \rho(t)] d t \\
+ & \sqrt{\kappa_{1}}\left[\hat{a}^{\dagger}, \rho(t)\right] \beta d t-\sqrt{\kappa_{1}}[\hat{a}, \rho(t)] \beta^{*} d t \\
- & \eta \kappa_{2}\left(\hat{a} \rho(t) \hat{a}^{\dagger}-\operatorname{Tr}\left(\hat{a} \rho(t) \hat{a}^{\dagger}\right) \rho(t)\right) d t \\
& +\left(\frac{\hat{a} \rho(t) \hat{a}^{\dagger}}{\operatorname{Tr}\left(\hat{a} \rho(t) \hat{a}^{\dagger}\right)}-\rho(t)\right) d \hat{N} \\
+ & \frac{1}{2} \kappa\left(-\hat{a}^{\dagger} \hat{a} \rho(t)-\rho(t) \hat{a}^{\dagger} \hat{a}+2 \hat{a} \rho(t) \hat{a}^{\dagger}\right) d t .
\end{aligned}
$$

$d \hat{N}$ is a stochastic variable, which assumes the value 1 with probability $\eta \kappa_{2} \operatorname{Tr}\left(\hat{a} \rho(t) \hat{a}^{\dagger}\right) d t$ and the value 0 with probability $1-\eta \kappa_{2} \operatorname{Tr}\left(\hat{a} \rho(t) \hat{a}^{\dagger}\right) d t$.

\section{APPLICATIONS}

\section{A. The empty cavity}

We first consider the time evolution of the state of the light field in the absence of any quantum system in the cavity. This situation leads to a coherent state cavity field because beam splitters transform coherent states into coherent states and because homodyne measurements also preserve the coherent state nature of the cavity field (see e.g. Ref. [20]). Inserting $H=0$ and $\rho(t)=C(t)|\xi(t)\rangle\langle\xi(t)|$ in Eq. (21), we obtain

$$
\frac{d \xi(t)}{d t}=-\frac{\kappa}{2} \xi(t)+\sqrt{\kappa_{1}} \beta,
$$

and if $\beta$ is time independent, which implies that the input light is on resonance with the cavity, $\xi(t)$ approaches $\xi=$ $2 \sqrt{\kappa_{1}} \beta / \kappa$ for $t \gg 2 / \kappa$.

We note that $\xi$ is a factor of $2 \sqrt{\kappa_{1}} /(\sqrt{\tau} \kappa)$ larger than the coherent state amplitude $\sqrt{\tau} \beta$ of a segment of the input field of length $c \tau$, and, in the limit of very weak coupling between the light field and a system, the presence of the cavity enhances the Rabi frequency of transitions between different states of the system by the same factor. For $\kappa_{3}=0$ and $\kappa_{1}=\kappa_{2}$ the factor reduces to $1 / t_{2}$, which equals the square root of twice the average number of round trips of a photon in the cavity in absence of the system and in absence of the input field. The factor of two appears due to destructive interference between the input field and the cavity field for $\xi(t)=2 \sqrt{\kappa_{1}} \beta / \kappa$, which ensures that no light is lost at beam splitter $\mathrm{BS}_{1}$ in the presence of the input light.

\section{B. Analytical solution for a simple system}

As a nontrivial application of the stochastic master equation we analyze the setup proposed in Ref. [4] to gen- erate Dicke states. Here the system consists of $N$ noninteracting identical atoms each with two ground state levels $|f\rangle$ and $|g\rangle$. The cavity field couples the level $|f\rangle$ to an exited level $|e\rangle$, and below it is assumed that the radiation is sufficiently off-resonant to avoid population of the exited level. In that case the interaction merely shifts the phase of the light field by an amount that is proportional to the number of atoms in the state $|f\rangle$, and since the homodyne detector is sensitive to the phase shift, the state of the monitored system slowly approaches an eigenstate of the operator $\hat{n}$, which counts the number of atoms in the state $|f\rangle$. For atoms all coupling with equal strengths to the light field, the relevant Hamiltonian reads

$$
H=\hbar g \hat{a}^{\dagger} \hat{a} \hat{n},
$$

where $g$ represents the strength of the coupling. We assume that the atoms are initially prepared in a state, which is symmetric under exchange of any two atoms, and we denote the symmetric state with $n$ atoms in the state $|f\rangle$ by $|n\rangle$. Since the Hamiltonian (28) preserves the symmetry under exchange of any two atoms, this allows us to use the restricted basis consisting of the states $|n\rangle$ with $n=0,1, \ldots, N$.

To simplify the stochastic master equation, we write the density operator of the system and the cavity mode as

$$
\rho(t)=\sum_{n=0}^{N} \sum_{m=0}^{N} \rho_{n m} \otimes|n\rangle\langle m|
$$

where $|n\rangle$ and $|m\rangle$ refer to the state of the system and $\rho_{n m}$ are time dependent cavity mode operators. Inserting this into Eq. (24), we obtain the $(N+1)^{2}$ independent linear equations

$$
\begin{aligned}
\frac{d \rho_{n m}}{d t}= & -i g\left(n \hat{a}^{\dagger} \hat{a} \rho_{n m}-m \rho_{n m} \hat{a}^{\dagger} \hat{a}\right) \\
& +\sqrt{\kappa_{1}}\left[\hat{a}^{\dagger}, \rho_{n m}\right] \beta-\sqrt{\kappa_{1}}\left[\hat{a}, \rho_{n m}\right] \beta^{*} \\
& -i \sqrt{\eta \kappa_{2}}\left(\hat{a} \rho_{n m} e^{-i \phi}-\rho_{n m} \hat{a}^{\dagger} e^{i \phi}\right) \frac{d \hat{y}}{d t} \\
& +\frac{1}{2} \kappa\left(-\hat{a}^{\dagger} \hat{a} \rho_{n m}-\rho_{n m} \hat{a}^{\dagger} \hat{a}+2 \hat{a} \rho_{n m} \hat{a}^{\dagger}\right) .
\end{aligned}
$$

To determine $\left\langle n\left|\rho_{\text {sys }}\right| n\right\rangle$, where $\rho_{\text {sys }}$ is the normalized density operator for the system, it is sufficient to solve Eq. (30) for $n=m$. If the initial state of the system is $|n\rangle\langle n|$, we can replace the operator $\hat{n}$ in the Hamiltonian (28) by the number $n$, whereby the system is effectively reduced to a phase shifter, and it follows that the cavity field is in a coherent state. Equation (30) for $n=m$ is, however, not mathematically different in the general case, and it is thus solved by

$$
\rho_{n n}=C_{n}(t)\left|\xi_{n}(t)\right\rangle\left\langle\xi_{n}(t)\right| .
$$

Inserting Eq. (31) into Eq. (30), we find

$$
\frac{d \xi_{n}(t)}{d t}=-\left(\frac{\kappa}{2}+i g n\right) \xi_{n}(t)+\sqrt{\kappa_{1}} \beta
$$


and

$$
d C_{n}(t)=\sqrt{\eta \kappa_{2}}\left(-i e^{-i \phi} \xi_{n}(t)+i e^{i \phi} \xi_{n}^{*}(t)\right) C_{n}(t) d \hat{y},
$$

and thus, for a time independent $\beta$,

$$
\xi_{n}(t)=\left(\xi_{n}(0)-\frac{2 \sqrt{\kappa_{1}} \beta}{\kappa+2 i g n}\right) e^{-(\kappa / 2+i g n) t}+\frac{2 \sqrt{\kappa_{1}} \beta}{\kappa+2 i g n}
$$

and

$$
\begin{aligned}
C_{n}(t)=C_{n}(0) \exp \left(-\int_{0}^{t} r_{n}\left(t^{\prime}\right) \frac{d \hat{y}\left(t^{\prime}\right)}{d t^{\prime}} d t^{\prime}\right. & \\
& \left.-\frac{1}{2} \int_{0}^{t} r_{n}\left(t^{\prime}\right)^{2} d t^{\prime}\right),
\end{aligned}
$$

where $r_{n}(t) \equiv \sqrt{\eta \kappa_{2}}\left(i e^{-i \phi} \xi_{n}(t)-i e^{i \phi} \xi_{n}^{*}(t)\right)$.

The general solution simplifies considerably if the detector is turned off $(\eta=0)$ until $\xi_{n}(t)$ has obtained its steady state value

$$
\xi_{n}=\frac{2 \sqrt{\kappa_{1}} \beta}{\kappa} \frac{1-2 i g n / \kappa}{1+4 g^{2} n^{2} / \kappa^{2}},
$$

since in that case $r_{n}\left(t^{\prime}\right)=r_{n}$ can be moved outside the integrals, and $C_{n}(t)$ depends only on the sum $\hat{y}$ of all the infinitesimal increments $d \hat{y}$. $\left\langle n\left|\rho_{\mathrm{sys}}\right| n\right\rangle$ does not change as long as $\eta=0$, and below we thus simply assume that $\xi_{n}(0)$ is the steady state value. We note that this shift is insignificant if the changes of the state of the system induced by the homodyne measurements take place on a time scale that is much longer than $\kappa^{-1}$, which is often the case, because the coupling strength $g$ of the offresonant interaction between the system and the cavity mode is typically very small.

\section{Probing as a state preparation tool}

We next consider in more detail how the measurement of the phase shift of the transmitted light can be used to prepare different types of quantum mechanical states.

\section{Dicke states}

To prepare a Dicke state we choose $\phi=0$ (corresponding to a measurement of the $p$-quadrature of the field), since it follows from Eq. (36) and the definition of $r_{n}$ that those measurements are most sensitive to the induced phase changes in the limit $2 g / \kappa \ll 1$ and $\beta=\beta^{*}$. In this case

$$
C_{n}(t)=C_{n}(0) \exp \left(-r_{n} \hat{y}-r_{n}^{2} t / 2\right),
$$

with

$$
r_{n}=\frac{8 \beta g n \sqrt{\kappa_{1} \kappa_{2} \eta}}{\kappa^{2}+4 g^{2} n^{2}}
$$
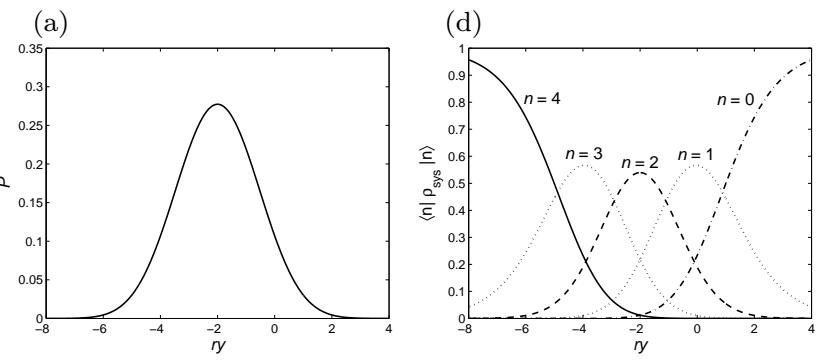

(b)

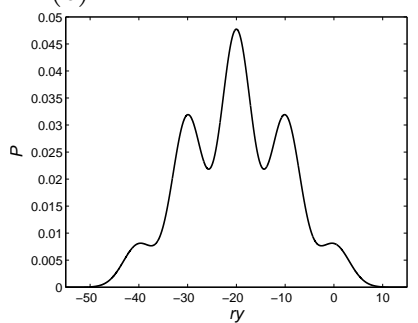

(e)

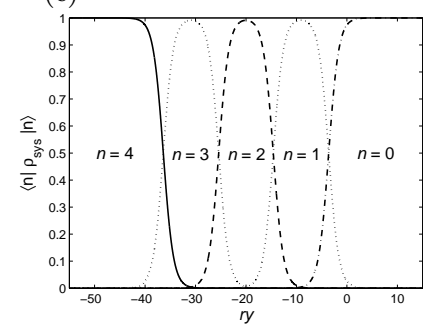

(c)

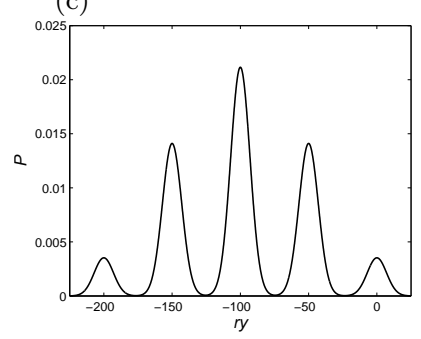

(f)

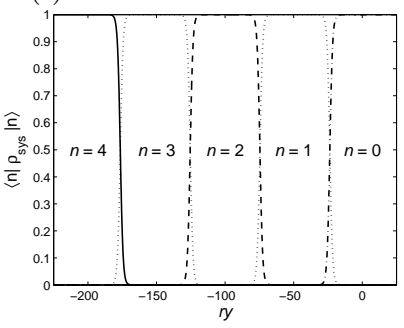

FIG. 3: (a)-(c): Probability density $P$ to measure the integrated signal $y$ in the interval from 0 to $t$ as a function of $y$ for $r^{2} t=1,10$, and 50, respectively. (d)-(f): $\left\langle n\left|\rho_{s y s}\right| n\right\rangle$ for $n=0,1,2,3,4$ as a function of $y$ for the same values of $r^{2} t$. The values of $C_{i}(0)$ are those obtained for the initial state $\left|\psi_{\text {sys }}\right\rangle=((|f\rangle+|g\rangle) / \sqrt{2})^{\otimes 4}$ of the system, and it is assumed that $2 g \ll \kappa$.

and, from Eq. (25), the state for which $\hat{y}$ assumes the value $y$ is obtained with probability density

$$
P=\sum_{n=0}^{N} \frac{C_{n}(0)}{\sqrt{2 \pi t}} \exp \left(-\frac{\left(y+r_{n} t\right)^{2}}{2 t}\right) .
$$

$P$ and the state preparation fidelity $\left\langle n\left|\rho_{\text {sys }}\right| n\right\rangle=$ $C_{n}(t) / \sum_{m=0}^{N} C_{m}(t)$ are plotted as functions of $y$ at different times for $C_{i}(0)=N ! / i ! /(N-i) ! / 2^{N}$ and $N=4$ in Fig. 3, neglecting the term $4 g^{2} n^{2}$ in the denominator of Eq. (38), such that $r_{n}=r n$, where $r$ is independent of $n$. Within this approximation $P$ consists of a sum of five Gaussians separated by $r t$ and of width $\sqrt{t}$, and the transition from overlapping Gaussians at small $r^{2} t$ to well separated Gaussians at large $r^{2} t$ is apparent in the figure. The plots of $\left\langle n\left|\rho_{\text {sys }}\right| n\right\rangle$ illustrate how the state of the system is gradually collapsed onto an eigenstate of the operator $\hat{n}$, and it is clear that each peak in $P$ corresponds to a specific value of $n$ if $r^{2} t$ is large. 


\section{Quantum superposition states}

It is also possible to use the setup to generate a superposition of two Dicke states as, for instance, the maximally entangled state $\left(|0\rangle+e^{i \theta}|N\rangle\right) / \sqrt{2}$. To do so we include an additional phase shift inside the cavity such that the Hamiltonian reads

$$
H=\hbar g \hat{a}^{\dagger} \hat{a}(\hat{n}-N / 2)
$$

With this Hamiltonian the states $|n\rangle$ and $|N-n\rangle$ are indistinguishable if the $x$-quadrature of the field is measured $(\phi=-\pi / 2$ in Eq. (20) $)$, and thus, for the initial state $\left|\psi_{\text {sys }}\right\rangle=((|f\rangle+|g\rangle) / \sqrt{2})^{\otimes N}$, a superposition of $|0\rangle$ and $|N\rangle$ is generated after sufficiently long measurement time with probability $1 / 2^{N-1}$. To achieve a pure atomic state, it is required to work under lossless conditions, i.e., under conditions where Eq. (22) is valid, and it is also important to avoid entanglement between the cavity field and the atoms at the final time, since the cavity field is to be traced out to obtain the atomic state. The last requirement is easily fulfilled by turning off the probe field at some point, letting the cavity field decay to the vacuum state while keeping the detector turned on. We note that the protocol is more demanding than the protocol to generate Dicke states due to the requirement of lossless conditions and because the difference between $r_{n}$ and $r_{n+1}$ is second order in $2 g / \kappa$, which leads to an increase in the required measurement time.

\section{Quantum Zeno effect and quantum jumps}

In the preceding subsections the state of the atoms was simply detected by an indirect continuous measurement, but we now add the complication of internal dynamics by allowing transitions between adjacent $|n\rangle$ states. Specifically, we consider the Hamiltonian

$$
H=\hbar g \hat{a}^{\dagger} \hat{a} \hat{n}+\hbar g_{s} \sum_{i=1}^{N}\left(\hat{\sigma}_{i,+}+\hat{\sigma}_{i,-}\right)
$$

where $\sigma_{i,+} \equiv|f\rangle_{i i}\langle g|$ and $\sigma_{i,-} \equiv|g\rangle_{i i}\langle f|$, and $i$ refers to atom number $i$, which, depending on the level structure of the atoms, may be realized by applying a microwave field propagating in a direction perpendicular to the cavity axis. Since

$$
\sum_{i=1}^{N} \hat{\sigma}_{i,+}|n\rangle=\sqrt{(n+1)(N-n)}|n+1\rangle,
$$

and

$$
\sum_{i=1}^{N} \hat{\sigma}_{i,-}|n\rangle=\sqrt{n(N+1-n)}|n-1\rangle
$$

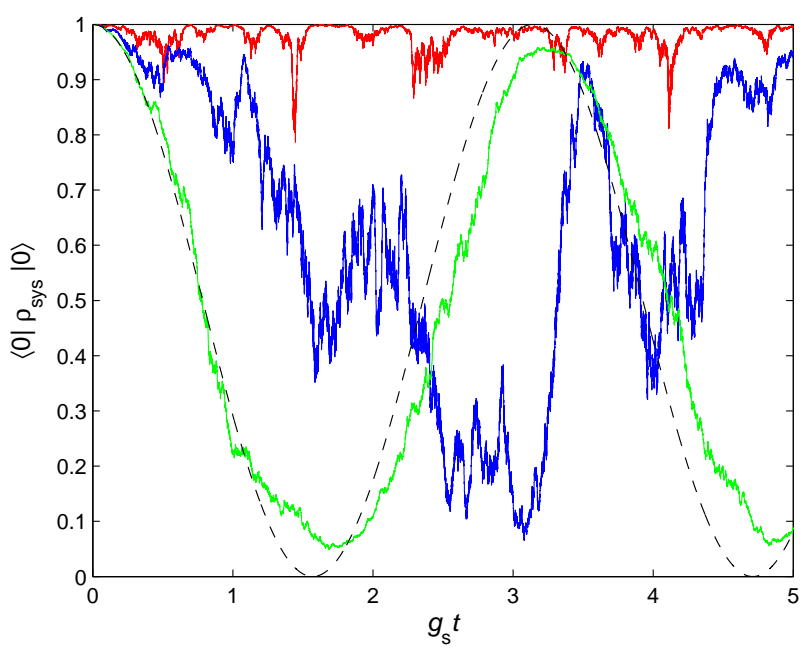

FIG. 4: $\left\langle 0\left|\rho_{\text {sys }}\right| 0\right\rangle$ as a function of scaled time $g_{\mathrm{s}} t$ for $N=1$, $\kappa_{1}=\kappa_{2}=0.5 \kappa, \kappa_{L}=0, \eta=1, \phi=0, g=0.2 \kappa$, $\beta=0.2 \sqrt{\kappa}, N_{p}=3$, and initial state $\rho(0)=|0\rangle|v a c\rangle\langle v a c|\langle 0|$, where $|v a c\rangle$ is the vacuum state of the cavity mode. For weak driving with $g_{s}=0.001 \kappa$ (red curve) the continuous measurements inhibit the coherent evolution between the states $|0\rangle$ and $|1\rangle$, while for strong driving with $g_{s}=0.05 \kappa$ (green curve) the time evolution of the state almost follows the deterministic evolution obtained for $g=0$ (dashed curve). The blue curve $\left(g_{s}=0.005 \kappa\right)$ illustrates the behavior of the state of the system in the intermediate regime. The same noise realization is used to compute all curves.

we obtain the stochastic master equation for the system and the cavity field by adding the terms

$$
\begin{aligned}
& -i g_{s} \sqrt{n(N+1-n)} \rho_{n-1, m} \\
& -i g_{s} \sqrt{(n+1)(N-n)} \rho_{n+1, m} \\
& +i g_{s} \sqrt{m(N+1-m)} \rho_{n, m-1} \\
& +i g_{s} \sqrt{(m+1)(N-m)} \rho_{n, m+1}
\end{aligned}
$$

to the right hand side of the master equation for the Hamiltonian in Eq. (28).

The added system Hamiltonian drives a coherent evolution between the different $|n\rangle$ states of the system, but if the measurements are sufficiently strong, the continuous back action of the measurements on the state of the system blocks the coherent evolution. To demonstrate this effect, which is known as the Quantum Zeno effect, we use the Milstein scheme (see Ref. [21]) to solve the nonlinear stochastic master equation numerically for a particular realization of the measurement outcomes. The accuracy of the integration may be checked for $g_{s}=0$ by application of the analytical results obtained in the previous subsection. We use the Fock state basis for the cavity mode and neglect terms in the density operator with more than $N_{p}$ photons in the cavity, where $N_{p} \gg 4 \kappa_{1}|\beta|^{2} / \kappa^{2}$ (see Sec. $\mathrm{VA}$ ). The results are shown in Fig. 4. Since $\left\langle 1\left|\rho_{\text {sys }}\right| 1\right\rangle$ is not exactly zero, there is a small probability that the system switches to state $|1\rangle$, 


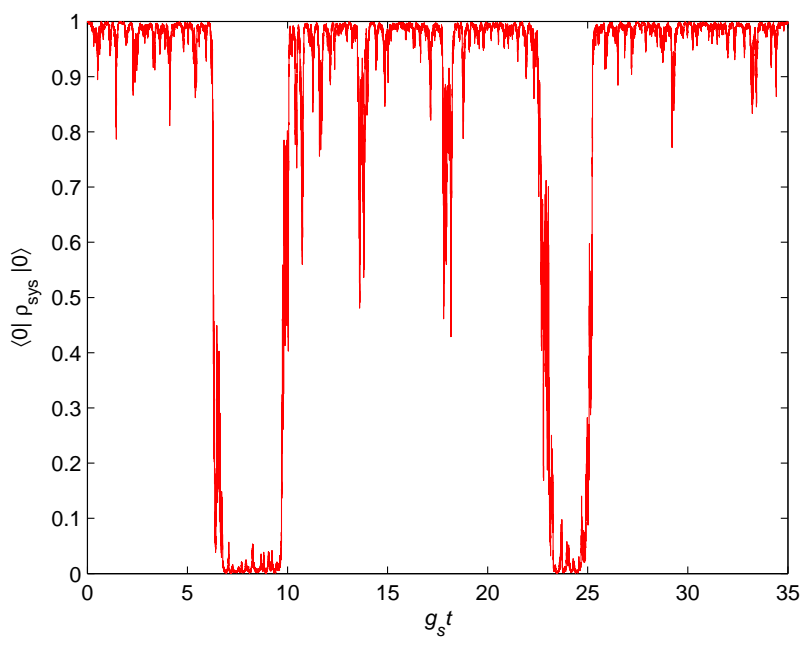

FIG. 5: Quantum jumps for a single atom in a cavity. The parameters are the same as in Fig. $4\left(g_{s}=0.001 \kappa\right)$.

where it is subsequently stabilized, and if the dynamics is integrated for sufficiently long time, several such quantum jumps between $|0\rangle$ and $|1\rangle$ will occur (Fig. (5).

We note that for general $N$ the states with $n=0$ or $n=N$ are easier to stabilize by measurements, since the square root factors in Eqs. (44), (45), (46), and (47) are smaller for $n$ close to 0 or $N$ than they are for $n$ close to $N / 2$.

Finally, as a simple example of feedback, we note that it is possible to generate a particular eigenstate of $\hat{n}$ by increasing $g_{s}$ whenever $\left\langle n\left|\rho_{\text {sys }}\right| n\right\rangle$ becomes small and decreasing $g_{s}$ to zero whenever $\left\langle n\left|\rho_{\text {sys }}\right| n\right\rangle$ becomes large.

\section{CONCLUSION}

In conclusion we have considered the influence of a specific indirect continuous measurement on the state of an abstract system, and we have derived the master equation determining the time evolution of the state of the system conditioned on the measurement outcomes. This equation provides a tool to analyze a wide variety of systems and phenomena in detail, and we have applied it to demonstrate the collapse of the state of a system onto an eigenstate of the measurement operator and to demonstrate quantum jumps and blocking of the internal dynamics of a system due to continuous measurements.

Indirect measurements are important because they constitute a special class of state manipulations that are very useful to prepare systems in specific quantum mechanical states. The crucial point is that the complete collapse of the state due to back action of a measurement performed directly on the system is avoided by performing instead the measurement on an auxiliary system that has interacted with the system. We showed examples with simple atomic level structures, but we emphasize that our analysis applies to quite general quantum systems and, for example, to multi-level atoms with shelving states leading to macroscopic quantum jumps in the transmitted intensity [22, 23]. A further step towards achieving control on the quantum state of a system is to apply feedback [24, 25, 26] by subjecting the system to disturbances that depend on the outcome of measurements. This situation may also be handled by the formalism developed in the present paper. The derived stochastic master equation is thus a valuable tool to investigate further state preparation protocols.
[1] A. Kuzmich, N. P. Bigelow, and L. Mandel Europhys. Lett. A 42, 481 (1998).

[2] L.-M. Duan, J. I. Cirac, P. Zoller, and E. S. Polzik, Phys. Rev. Lett. 85, 5643 (2000).

[3] H. L. Partner, B. D. Black, and JM Geremia, quantph/0708.2730.

[4] A. S. Sørensen and K. Mølmer, Phys. Rev. Lett. 91, 097905 (2003).

[5] S. Gleyzes, S. Kuhr, C. Guerlin, J. Bernu, S. Deléglise, U. B. Hoff, M. Brune, J.-M. Raimond, and S. Haroche, Nature (London) 446, 297 (2007).

[6] K. Jacobs and D. A. Steck, Contemporary Physics 47, 279 (2006).

[7] G. Lindblad, Commun. Math. Phys. 48, 119 (1976).

[8] R. J. Thompson, G. Rempe, and H. J. Kimble, Phys. Rev. Lett. 68, 1132 (1992).

[9] G. Rempe, R. J. Thompson, R. J. Brecha, W. D. Lee, and H. J. Kimble, Phys. Rev. Lett. 67, 1727 (1991).

[10] P. W. H. Pinkse, T. Fischer, P. Maunz, and G. Rempe, Nature (London) 404, 365 (2000).

[11] P. Horak, H. Ritsch, T. Fischer, P. Maunz, T. Puppe, P. W. H. Pinkse, and G. Rempe, Phys. Rev. Lett. 88, 043601 (2002).
[12] J. A. Sauer, K. M. Fortier, M. S. Chang, C. D. Hamley, and M. S. Chapman, Phys. Rev. A 69, 051804(R) (2004).

[13] K. M. Fortier, S. Y. Kim, M. J. Gibbons, P. Ahmadi, and M. S. Chapman, Phys. Rev. Lett. 98, 233601 (2007).

[14] M. Trupke, J. Goldwin, B. Darquié, G. Dutier, S. Eriksson, J. Ashmore, and E. A. Hinds, Phys. Rev. Lett. 99, 063601 (2007).

[15] A. Öttl, S. Ritter, M. Köhl, and T. Esslinger, Phys. Rev. Lett. 95, 090404 (2005).

[16] F. Brennecke, T. Donner, S. Ritter, T. Bourdel, M. Köhl, and T. Esslinger, Nature (London) 450, 268 (2007).

[17] Y. Colombe, T. Steinmetz, G. Dubois, F. Linke, D. Hunger, and J. Reichel, Nature (London) 450, 272 (2007).

[18] H. M. Wiseman and G. J. Milburn, Phys. Rev. A 47, 642 (1993).

[19] A. E. B. Nielsen and K. Mølmer, Phys. Rev. A 76, 033832 (2007).

[20] L. B. Madsen and K. Mølmer: Continuous measurements on continuous variable quantum systems: The Gaussian description, in "Quantum Information with Continuous Variables of Atoms and Light", Eds. N. Cerf, G. 
Leuchs, and E. S. Polzik. Imperial College Press, 2007; quant-ph/0511154

[21] P. E. Kloeden and E. Platen, Numerical Solution of Stochastic Differential Equations, Springer-Verlag, 1992.

[22] J. Metz and A. Beige, Phys. Rev. A 76, 022331 (2007).

[23] J. Metz, C. Schön, and A. Beige, Phys. Rev. A 76, 052307 (2007).
[24] D. A. Steck, K. Jacobs, H. Mabuchi, T. Bhattacharya, and S. Habib, Phys. Rev. Lett. 92, 223004 (2004).

[25] JM Geremia, Phys. Rev. Lett. 97, 073601 (2006).

[26] A. Negretti, U. V. Poulsen, and K. Mølmer, Phys. Rev. Lett. 99, 223601 (2007). 